\title{
¿Existe el Plain Spanish? La modernización del discurso jurídico-administrativo y su influencia en la traducción jurídica
}

\section{(Does the Plain Spanish exist? The Modernization of Legal and Administrative Discourse and its Impact on Legal Translation)}

CRistina Toledo BÁEZ

Universidad de Málaga

Fecha de recepción: 13 de mayo de 2011

Fecha de aceptación: 11 de julio de 2011

Resumen: En el presente artículo investigamos acerca del discurso jurídico-administrativo y sus transformaciones. Tras determinar las características terminológicas, morfológicas y sintácticas de dicho discurso, analizaremos el impacto que, sobre todo en Reino Unido y en Estados Unidos, ha tenido un movimiento de gran calado denominado Plain English, mediante el cual el ciudadano medio exige un lenguaje claro y comprensible por parte de la Justicia y de la Administración Pública. Mostraremos que este movimiento se ha extendido a nuestro país y estudiaremos las distintas medidas nacionales ofrecidas con el fin de lograr una comunicación eficaz con el público en general. Analizaremos la influencia de este lenguaje claro en la traducción jurídica mediante el análisis de una sentencia en las lenguas española e inglesa en aras a comprobar la viabilidad de un Plain Spanish.

Palabras clave: Plain Spanish. Discurso jurídico-administrativo. Traducción jurídica. Traducción.

Abstract: The paper examines the recent transformation and evolution of the administrative and legal language. After pointing out the main terminological, morphological and syntactical features of legal English, we analyse the impact that a movement called 'Plain English' has had, above all, in the UK and the USA. This movement demands the use of a clear and comprehensible language when the government or the administration communicates with the general public. We explore how this movement has also entered Spain by focusing on the initiatives taken by the Spanish government in order to achieve a plain, straightforward language in all legal and bureaucratic documents. We analyze the impact that this plain language is having on legal translation by comparing a British judicial sentence with a Spanish one. The possibility of having a 'Plain Spanish' is then considered as well as its potential impact.

Key words: Plain Spanish. Legal and Administrative Discourse. Legal Translation. Translation. 


\section{INTRODUCCIÓN}

La comunicación entre el Estado y los ciudadanos constituye un pilar básico de cualquier sociedad democrática. El derecho a acceder a una información clara y completa debe ser una garantía por parte de la Justicia y de la Administración Pública, ya que de ellas dependen asuntos legales y administrativos de vital importancia para el funcionamiento de un país.

A pesar de ello, el discurso jurídico-administrativo, tanto en la lengua española como en la inglesa, se caracteriza por la rigidez de su estructura y por un léxico muy conservador, lleno de tecnicismos y fijado de antemano a través de fórmulas y frases hechas. Por consiguiente, se trata de un sublenguaje complejo y difícil, motivo por el cual surgieron en diversos países de habla inglesa varios movimientos de ciudadanos que solicitaban claridad y transparencia en el lenguaje de la ley y de la administración de la justicia, como, por ejemplo, el Plain English Campaign ${ }^{1}$. En efecto, en el mundo anglosajón el ciudadano medio ha comprendido que debe exigir una redacción que le permita la comprensión total de los documentos legales ya que "la ignorancia de las leyes no excusa de su cumplimiento"; de esta forma, en el Reino Unido y en Estados Unidos los movimientos de gran calado que abogan por un lenguaje claro y sencillo en el ámbito jurídico y legal y que, incluso, han logrado, en el caso de Gran Bretaña, la simplificación del discurso procesal de la Ley de Enjuiciamiento Civil de 1998 (The Civil Procedure Rules 1998), se han extendido también a otros países como Francia, Suecia, Italia y España. Así, en nuestro país, una de las iniciativas de mayor trascendencia es el Plan de Transparencia Judicial de 2005, el cual preconiza la actuación primordial de "ofrecer a los ciudadanos una justicia comprensible, con un lenguaje inteligible". Sin embargo, ¿se plasma este objetivo en la redacción de documentos jurídicos o resoluciones judiciales que atañen directamente al ciudadano no versado en materias legales?

En consonancia con lo anterior, analizaremos en este artículo dos sentencias de ordenamientos jurídicos diferentes, a saber, el británico y el español, en aras a determinar si el Plain English se traduce, en el sentido literal de la palabra, en un verdadero Plain Spanish acorde con las máximas de claridad y accesibilidad en las relaciones Estado-ciudadano. De este modo, estudiaremos la simplificación y, por ende, modernización del discurso jurídico-administrativo en la traducción jurídica y comprobaremos cómo influye en todo el proceso traductológico.

\footnotetext{
${ }^{1}$ Destacamos la existencia de páginas web especializadas en el Plain English Campaign como es el caso de <http://www.plainenglish.co.uk/>.
} 


\section{PLAIN ENGLISH: ORIGEN, EVOLUCIÓN Y APLICACIÓN}

La Administración contemporánea ha prestado especial atención al proceso de "modernización" del lenguaje administrativo; se entiende por tal la necesidad de proceder al cambio del modelo tradicional de "Administración separada", marcadora de las distancias que se establecen entre ella y los administradores, sustituyéndola por un tipo de Administración más abierto e igualitario, vuelto hacia el exterior y para el cual se consideran de especial importancia la información y las comunicaciones con el entorno (M. A. P. 1990: 29). Sobre estos principios se puede constatar, en muchos países, la existencia de un proceso de renovación del lenguaje jurídico-administrativo. Algunos de estos factores que impulsan un proceso renovador de este tipo son los siguientes: la demanda social en defensa de una mayor comprensión en los documentos jurídicos; el factor económico, en particular a lo que atañe a la reducción del volumen de documentación y, por último, la modernización de la imagen institucional de la Administración Pública (M. A. P. 1990: 29-30).

Este proceso de modernización se articula en la década de los setenta prácticamente en todos los países de nuestro entorno. En realidad, es en los países de habla inglesa donde ha cumplido una función relevante el denominado Plain English Campaign (1996) con el que se designa una tendencia al uso de un lenguaje sencillo e inteligible en la documentación oficial (Etxebarría, 1997: 366). Este plan se articula en los años setenta y su fundadora, Chrissie Maher, se propuso combatir el oscuro e ininteligible lenguaje que se utilizaba en la Administración británica. Así, de acuerdo con la distinción de Alcaraz Varó et alii (2001: 91) entre, por un lado, officialese o jerga burocrática empleada por la Administración y, por otro, legalese o la jerga utilizada por los juristas, el Plain English Campaign comenzó luchando contra el primero de ellos, pues el ciudadano medio tenía dificultades con el lenguaje que se encontraba, por ejemplo, al rellenar el impreso de la declaración de la renta. No obstante, el legalese también se ha visto modificado, ya que, de hecho, el Plain English Campaign ha influido a todas luces en la simplificación del lenguaje procesal de la Ley de Enjuiciamiento Civil de 1998 (Civil Procedures Rules 1998) del Reino Unido (Alcaraz Varó et alii 2001).

Las líneas generales sobre las que se articula el plan de simplificación del inglés jurídico son, de acuerdo con Extebarría (1997: 366), las siguientes:

- Uso de un lenguaje apropiado al mensaje y a los destinatarios de tal modo que la información resulte accesible.

- Utilización de un lenguaje cotidiano, de tipo conversacional.

- Uso de sintaxis y de frases cortas.

- Empleo de formas verbales en voz activa. 
- Elección de formas de tratamientos de primera y segunda persona.

- No utilización de extranjerismos ni tecnicismos.

- Definición del mensaje con el fin de lograr una mayor eficacia comunicativa y de ofrecer la información de la forma más clara y eficaz posible.

- Utilización de léxico estándar.

El Plain English Campaign alcanzó gran magnitud en Estados Unidos, de tal forma que el Presidente Carter desarrolló una Orden del Poder Ejecutivo (la Executive Order 12044), la cual determinaba que toda norma emanada de la Administración tenía que estar "estar redactada en un lenguaje sencillo y comprensible al menos para todos aquellos que deban cumplirla" ${ }^{2}$. Hoy día, todos los textos legislativos y administrativos que se publican a través del Federal Register, que comprende la compilación de toda la legislación federal del país, han de ajustarse a normas precisas de forma y contenido que informen al lector no experto en la materia y logren llegar al administrado sin trabas lingüístico-discursivas. Los textos se caracterizan por un lenguaje sencillo con subtítulos clarificadores y una organización lógica del discurso (Comet i Codina, 1986: 28-29) ${ }^{3}$.

A tenor de las iniciativas citadas en las páginas precedentes, queda demostrada la influencia y aplicación práctica de la idea de la simplificación del discurso jurídico-administrativo. No obstante, esta idea de simplificación no está exenta de detractores, como es el caso de letrados y juristas que esgrimen multitud de razones para conservar la singularidad del inglés jurídico. Las tres más importantes son: a) las garantías jurídicas que a todos ofrece este lenguaje especializado; b) su propio dinamismo, que asegura su adaptación y modernidad, y c) el paralelismo constante entre este lenguaje especializado y el coloquial o corriente, que se encargan de poner de relieve los comentaristas, a fin de que los legos en la materia puedan comprender los hechos jurídicos (Alcaraz Varó, 2001: 73). Además, tal y como señalan Alcaraz Varó et alii (2001: 91) y Solan (1993: 135), se ha comprobado que muchos documentos jurídicos redactados con el inglés general tampoco son comprendidos por el ciudadano medio, dificultad que se debe a la complejidad de los conceptos y no al oscurantismo del lenguaje.

\footnotetext{
${ }^{2}$ Reproducimos a continuación la cita original: "The regulation is written in plain English and is understandable to those who must comply with it". La Executive Order 12044 se encuentra disponible en la URL <http://www.presidency.ucsb.edu/ws/index.php?pid=30539>.

${ }^{3}$ En cuanto a la modernización del lenguaje jurídico-administrativo en otros países europeos, hemos de destacar en particular la relevancia que ha adquirido en Suecia, Italia y Francia, $s$ bien no nos detendremos en sus características en el presente artículo (cf. Toledo Báez, 2008 y 2009).
} 
A pesar de estas críticas, si estudiamos todas las medidas adoptadas por los gobiernos de diversos países, podemos llegar a considerar como justa y necesaria esta simplificación y, a la postre, modernización del discurso jurídico-administrativo, particularmente cuando se trata de comunicarse con el ciudadano medio. De esta forma, como botón de muestra de un uso del Plain English destacamos que en la fase procesal llamada Charge to jury ("instrucciones al jurado") los jueces utilizan un inglés claro y sencillo, a la par que jurídico, para orientar al jurado sobre la doctrina jurídica que es de aplicación en el proceso en curso (Alcaraz Varó et alii, 2001: 92).

Con sus luces y sus sombras, la simplificación y modernización del discurso jurídico-administrativo va ganando terreno paulatinamente en el mundo anglosajón y en nuestros países vecinos (cf. Toledo Báez, 2008). Ante esto, cabe preguntarse cuál es la situación en España y qué medidas se han tomado para agilizar el discurso entre la justicia y la Administración y el ciudadano; todo ello será objeto de análisis en el siguiente apartado de nuestro artículo.

\section{EL SUBLENGUAJE JURÍDICO-ADMINISTRATIVO EN ESPAÑA Y SU MODERNIZACIÓN}

Tal y como señalan numerosos estudiosos del discurso jurídico-administrativo español (cf. Alcaraz Varó y Hughes, 2001; Martín et alii, 1996; Toledo Báez, 2009 y 2010, entre otros) constituye éste un sublenguaje complicado, oscuro la mayoría de las veces y ajeno al ciudadano medio. Así, por medio de este discurso, la democratización de la Administración y la Justicia y su cercanía a la ciudadanía distan de estar próximas. No obstante, en nuestro país han existido diversas iniciativas que abogan por un proceso de modernización y simplificación del sublenguaje del Derecho y la Administración, de las cuales destacamos, en particular, tres: la aparición de la Ley de Procedimiento Administrativo de 1958; la publicación de Calvo Ramos, Introducción al estudio del lenguaje administrativo, de 1980; y, la más relevante de todas ellas, la publicación del Manual de estilo del lenguaje administrativo (M. A. P. 1990).

En cuanto a las propuestas de acción para lograr la modernización de este tipo de discurso, Prieto (1991:134-141) las resume de la siguiente forma: 1) elaborar un Plan de mejora de lenguaje jurídico-administrativo castellano; 2) limitar la acción del legislador en la normalización del uso del lenguaje administrativo; 3 ) introducir la quietud comunicativa y lingüística en los procesos de redacción de las normas administrativas de carácter general. De las tres propuestas, sin duda la primera y la tercera encarnan la modernización y simplificación del sublenguaje jurídico-administrativo español en aras a alcanzar la perfecta simbiosis entre lenguaje jurídico y democracia. Llegados a este punto, cabe señalar que, hasta la fecha, han 
sido precisamente las comunidades autónomas con lengua territorial propia los artífices de los cambios más renovadores ya que, apoyándose en el marco constitucional, han emprendido una acción modernizadora más vigorosa de sus lenguajes administrativos (M. A. P., 1990: 32).

No obstante, el gobierno central comienza a percibir la necesidad de cambios en las relaciones Estado-ciudadano. En este contexto, el 16 de abril de 2002 se aprobó el Pacto de Estado para la Reforma de la Justicia, que cuenta entre sus principales aportaciones con la adopción por unanimidad en el Congreso de los Diputados de la Carta de Derechos de los Ciudadanos ante la Justicia. Como parte de dicho Pacto, en 2005 y mediante la resolución de 28 de octubre de 2005 (Boletín Oficial del Estado de 1 de noviembre de 2005), se aprobó el Plan de Transparencia Judicial, el cual constituye un ambicioso proyecto destinado a conseguir una mayor transparencia de los procedimientos judiciales y, con este fin, se lleva a cabo una serie de actuaciones primordiales, entre las que destacan, sobre todo (BOE de 1 de noviembre de 2005):

- Poner en funcionamiento Oficinas de Atención al Ciudadano que le permitan recibir información general y actualizada sobre el funcionamiento de juzgados y tribunales.

- Ofrecer a los ciudadanos un servicio público judicial de mayor agilidad, calidad y eficacia, a través de métodos de organización e instrumentos procesales más modernos.

- Facilitar a los ciudadanos información continua y transparente sobre el estado de los asuntos tramitados y pendientes, así como el contenido actualizado de las leyes españolas y de la Unión Europea.

- Ofrecer a los ciudadanos una justicia comprensible, con un lenguaje inteligible, tanto en las notificaciones, citaciones, emplazamientos y requerimientos como en las sentencias y demás resoluciones judiciales.

Sin lugar a dudas, en el aspecto lingüístico que nos ocupa, la última actuación constituye la de mayor interés pues supone una modificación profunda del discurso jurídico-administrativo, de forma que casi podríamos hablar de un Plain Spanish, pues éste ha de ser comprensible para todos de forma que cualquier ciudadano pueda entender sin mucha dificultad los documentos que le transmiten información. Esto cobra aún mayor importancia en el caso de documentos que exigen una actuación por parte del ciudadano de a pie, como, puedan ser, por ejemplo, las citaciones, los emplazamientos y las sentencias $u$ otras resoluciones judiciales. En cuanto a las sentencias en particular, la Carta de Derechos de los Ciudadanos ante la Justicia, anteriormente señalada, dispone en el apartado número 7 que 
"el ciudadano tiene derecho a que las sentencias y demás resoluciones judiciales se redacten de tal forma que sean comprensibles por sus destinatarios, empleando una sintaxis y estructura sencillas, sin perjuicio de su rigor técnico" (Carta de Derecho de los Ciudadanos ante la Justicia, 2005:3) ${ }^{4}$. Por tanto, queda claro el deseo de la justicia en tanto comunicadora con los ciudadanos en la claridad de los documentos que instan al cumplimiento de una acción, como puede ser una sentencia. Pero, ¿es esto realmente cierto? ¿El lenguaje empleado en la redacción de una sentencia es sencillo y comprensible para el ciudadano medio? Daremos cuenta de ello en el apartado siguiente.

3 INFLUENCIA DE LA SIMPLIFICACIÓN DEL DISCURSO JURÍDICO-ADMINISTRATIVO ESPAÑOL EN LA TRADUCCIÓN JURÍDICA: ESTUDIO DE DOS SENTENCIAS

A continuación, analizaremos dos sentencias, una en español y otra en inglés como ejercicio previo a una traducción pedagógica en aras a determinar si el Plain English promulgado por la justicia británica se traduce, en el sentido literal de la palabra, en un Plain Spanish.

\subsection{Las sentencias en el Reino Unido: análisis de un ejemplo}

En el Reino Unido, como ya hemos indicado anteriormente (cf. apartado 3), el Estado es plenamente consciente de la necesidad de ofrecer un lenguaje sencillo y comprensible, tal y como se demostró con la reforma de la Ley de Enjuiciamiento Civil de 1998, en la cual primó la simplificación mediante la reducción de tecnicismos. Dicha simplificación ha alcanzado también los tribunales, tanto civiles como penales, y una muestra de ello es la creación en 2004 de un Consejo para las normas condenatorias $^{5}$ (Sentencing Guidelines Council), cuyo objetivo primordial es el de guiar a los tribunales de Inglaterra y Gales a la hora de dictar sentencias en casos de todo tipo. Así, en palabras del propio Consejo, "the purpose of this Council is to promote consistency in sentencing by providing clarity for courts, court users and victims so that everyone knows what to expect" (Theft and Burglary Guideline ${ }^{6}$ 2008: 6).

En consecuencia, la simplificación del discurso jurídico-administrativo en Reino Unido modifica el lenguaje y la estructura de los documentos elaborados por los tribunales. Con el fin de comprobar si verdaderamente se ha logrado dicha simplificación, analizaremos un fragmento de una

\footnotetext{
${ }^{4}$ Puede consultarse la Carta de Derechos de los Ciudadanos ante la Justicia en la siguiente dirección:<http://www.juecesdemocracia.es/ActualidadMJU/2006/marzo/cartaderechos\%5B1\%5 D.pdf $>$.

${ }_{6}^{5}$ La traducción es nuestra.

${ }^{6}$ Proponemos traducir Theft and Burglary Guideline como Directrices en caso de hurto o robo.
} 
sentencia, en concreto una sentencia del High Court of Justice por un caso de fraude:

Lord Justice Moses:

Introduction

1. This is the judgment of the Court.

2. Between 30 July 2004 and 14 December 2006 a team of Serious

Fraud Office lawyers, accountants, financial investigators and police officers carried out an investigation into allegations of bribery by $B A E$ Systems plc (BAE) in relation to the Al-Yamamah military aircraft contracts with the Kingdom of Saudi Arabia. On 14 December 2006 the Director of the Serious Fraud Office announced that he was ending the SFO's investigation.

$[\ldots]$

Facts

8. Since this case has aroused public concern, we should stress that which is well-recognised in the field of public law. This court is not concerned to conduct an enquiry into the facts which led to the Director's decision, save to the extent necessary to reach a conclusion as to whether that decision was lawful. The defendant has disclosed facts which are sufficient for the purpose of reaching a conclusion but they are not comprehensive and it is no part of the court's function in these judicial review proceedings to achieve a more complete account of the events, unless omission inhibits a correct legal conclusion. We emphasise that, through the efforts of Treasury Counsel and those by whom he is assisted, there has been sufficient disclosure to enable us to reach a solution to the essential question whether the Director acted lawfully. We turn, then, to the facts on which the court needs to rely.

[...]

Conclusion

170. The claimants succeed on the ground that the Director and Government failed to recognise that the rule of law required the decision to discontinue to be reached as an exercise of independent judgment, in pursuance of the power conferred by statute. To preserve the integrity and independence of that judgment demanded resistance to the pressure exerted by means of a specific threat. That threat was intended to prevent the Director from pursuing the course of investigation he had chosen to adopt. It achieved its purpose.

Una vez expuesto este breve fragmento de la sentencia, cabe señalar que sus características más relevantes son las siguientes:

- La macroestructura del texto es clara, dividida en secciones (introduction, facts y conclusions, entre otras) y numeradas, lo cual facilita en gran medida la lectura y, por extensión, la comprensión de la sentencia. 
- Escasa aparición de la voz pasiva, pues en las partes seleccionadas como ejemplos aparece sólo en dos oraciones ("[...] that the rule of law required the decisión to discontinue to be reached; that thread was intented to prevent [...]"). De este modo, se sigue una de las recomendaciones del Plain English (cf. apartado 3) de utilizar la voz activa en la medida de lo posible.

- Repeticiones de ciertos elementos por mor de la claridad ("On 14 December 2006 the Director of the Serious Fraud Office announced that he was ending the SFO's investigation").

- Empleo de un lenguaje no excesivamente complicado, si bien tampoco se puede clasificar como sencillo. En todo caso, su comprensión no entraña gran dificultad, aunque la parte de las conclusiones, en concreto el fragmento "in pursuance of the power conferred by statute. To preserve the integrity and independence of that judgment demanded resistance to the pressure exerted by means of a specific threat" presenta mayor complicación sintáctica.

En resumen, comprobamos que, en el caso de nuestra sentencia objeto de estudio, en la mayor parte del texto se hace uso de un lenguaje comprensible y no innecesariamente enrevesado. Por tanto, es patente que el Plain English cobra vida en los documentos jurídicos británicos.

\subsection{Las sentencias en España: análisis de un ejemplo}

Una vez comprobada la aplicación de un lenguaje sencillo en una sentencia británica, analizaremos a continuación una sentencia española con el objetivo de comprobar si cumple las indicaciones de claridad e inteligibilidad por las que aboga el Plan de Transparencia Judicial. La sentencia que aparece a continuación es objeto de análisis en un artículo de Rodríguez Achútegui (2006: 7) y proviene de un Juzgado de Instancia que condena a un supermercado y a su aseguradora a abonar una cantidad determinada, sin costas, a una señora que sufrió un accidente en su interior. La aseguradora recurre y la respuesta judicial que obtiene es la siguiente:

F.J. UNICO: "A las consideraciones de la instancia, las cuales hacemos nuestras, afirmando responsabilidad de la codemandada "Supermercado S. SA» con traslado de obligación indemnizatoria a su compañía de Seguros «B.P.S.R. SA», con toma de decisión basada en análisis de la culpa desde un punto de vista cuasi objetivo, obligando a indemnizar a aquel que saca un provecho derivado de actividad empresarial, bajo la premisa de ocurrencia de accidente en la propiedad donde desarrolla el negocio y siempre que no exista culpa exclusiva de la víctima, añadir, que en el presente caso no sólo existe obligación 
resarcitoria por la teorización expuesta, sino que es tangible negligencia en el proceder de la demandada. Literalizando documental obrante en la alzada conteniendo carta de la codemandada "Supermercado S. SA» a su correduría de Seguros, en relación con el accidente objeto de análisis, «esta señora se tropezó con unas cajas de mercancías que había comprado otro cliente, justo a la salida de nuestra escalera mecánica», uniendo la testifical de doña S.R.A., contestación a la repregunta cuarta «presenció el accidente, vio cómo pasaba la niña y que la señora no podía pasar y se cayó», es afirmable manifestar negligencia en la demandada al no tener en perfectas condiciones de utilización la vía de salida del establecimiento. Siendo la escalera mecánica, vía de salida, de titularidad propia, siendo el objeto, caja de mercancías, que obstaculiza el final de la precitada escalera propiedad de la demandada siendo manifiesto que dicha vía debe estar expedita, apta para su utilización como salida del centro comercial, no produciéndose tal realidad, se plasma negligencia, tangibilizada en la falta de actuación de operario para retirar todo obstáculo que impida la salida, y no sólo la impida sino que cree riesgo para las personas, materializado en la presente, en el accidente analizado, base de la reclamación. [...]

Por lo expuesto y lo reflejado en la instancia, se desestima el recurso, ratificándose la resolución recurrida e imponiéndose a la apelante las costas de la presente alzada por mor del párrafo 2 del art. 710 de la LECiv".

A todas luces, este fragmento de sentencia constituye un ejemplo claro de la falta de aplicación de un lenguaje claro y sencillo para el ciudadano medio. Su estructura confusa, su lenguaje farragoso y su falta de claridad lo convierten en el extremo opuesto de lo que se pretende alcanzar en el Plan de Transparencia Judicial. Con el fin de señalar las carencias más patentes de la sentencia, analizaremos sus características:

1. Estructura complicada y confusa. La macroestructura de la sentencia carece de organización y coherencia, pues no se emplea la estructura clásica de una sentencia española, es decir, los apartados antecedentes de hecho, fundamentos de derecho y el fallo. Tal y como señala Rodríguez Achútegui (2006), si bien la regulación legal es tajante al exigir párrafos separados y numerados (art. 248,3 de la Ley Orgánica $6 / 1985$, de 1 de julio, del Poder Judicial, LOPJ), lo habitual es que antecedentes de hecho y, sobre todo, fundamentos de hecho sean muy extensos, empleen frases subordinadas y huyan del punto y aparte y punto y seguido. No obstante, en el ejemplo antes expuesto se aprecia claramente este defecto, ya que se emplea la forma de Fundamento Jurídico Único, en lugar de separar el pronunciamiento principal, que consta en el primer párrafo, del pronunciamiento en costas, que debiera estar en otro diferente. Por tanto, hubiera sido más razonable o bien separar los párrafos tras cada punto y seguido, permitiendo descansar al lector y apreciar cada idea por 
separado, o bien seguir a rajatabla la previsión legal y numerar cada párrafo como un fundamento jurídico. El resultado de estas correcciones se detallará en páginas posteriores.

2. Expresiones incorrectas además de ortotipografía y sintaxis mal empleadas. Otra de las características más evidentes de la sentencia es el frecuente empleo del gerundio, forma impersonal que si no se emplea con exactitud gramatical provoca imprecisión y, por lo tanto, falta de rigor jurídico. Ejemplo claro es la oración que comienza con literalizando, lo cual indica que se recoge literalmente el contenido de un documento; otro ejemplo aparece en el tercer párrafo, que comienza hasta en tres ocasiones la frase con siendo, que podría cambiarse por «al ser», «por ser» u otra expresión semejante. Por otro lado, cabe señalar la omisión o inclusión errónea de los artículos definidos, pues, por ejemplo, encontramos «es tangible negligencia» en lugar de «es tangible la negligencia» o «es afirmable manifestar negligencia», cuando podría aparecer «se puede apreciar la negligencia». No obstante, uno de los errores más obvios de la sentencia es su mal uso de la ortotipografía, pues la puntuación equivocada da lugar a una dificultad de comprensión. En este caso, en el párrafo tercero, el uso de una coma agilizaría la lectura y facilitaría la comprensión de la siguiente oración: «que obstaculiza el final de la precitada escalera propiedad de la demandada siendo manifiesto que dicha vía debe estar expedita». Si tras demandada se emplease una coma, la oración tendría sentido porque se ha comenzado con un siendo, que luego se reitera en la frase yuxtapuesta, que tendría su tercera expresión enfática en el nuevo gerundio. En todo caso, en cuanto a la sintaxis, es recomendable hacer gala de simplicidad y emplear oraciones cortas, en estilo directo, que faciliten la comprensión del texto y eviten la perplejidad del lector.

3. Léxico incorrecto o de difícil comprensión. Tal y como apunta Rodríguez Achútegui (2006: 10), en ocasiones «el jurista decide invadir el campo del académico y con absoluta naturalidad inventa palabras inexistentes en castellano». Tal es el caso de obrante, recogida en la sentencia y muy empleada, o tangibilizar o afirmable, que no figuran en el Diccionario de la Real Academia Española de la Lengua.

En definitiva, tras haber detallado las modificaciones que facilitarían la comprensión de la sentencia, procedemos a comparar en la tabla siguiente la sentencia original junto con la sentencia modificada de acuerdo con los cambios anteriormente propuestos ${ }^{7}$ :

\footnotetext{
${ }^{7}$ En el caso de la sentencia modificada, en cursiva aparecen los cambios, entre corchetes los cambios de ubicación y entre paréntesis lo suprimido.
} 


\begin{tabular}{|c|c|}
\hline Sentencia original & Sentencia modificada \\
\hline $\begin{array}{l}\text { F.J. UNICO: "A las consideraciones } \\
\text { de la instancia, las cuales hacemos } \\
\text { nuestras, afirmando responsabilidad } \\
\text { de la codemandada "Supermercado } \\
\text { S. SA» con traslado de obligación } \\
\text { indemnizatoria a su compañía de } \\
\text { Seguros «B.P.S.R. SA», con toma de } \\
\text { decisión basada en análisis de la } \\
\text { culpa desde un punto de vista cuasi } \\
\text { objetivo, obligando a indemnizar a } \\
\text { aquel que saca un provecho } \\
\text { derivado de actividad empresarial, } \\
\text { bajo la premisa de ocurrencia de } \\
\text { accidente en la propiedad donde } \\
\text { desarrolla el negocio y siempre que } \\
\text { no exista culpa exclusiva de la } \\
\text { víctima, añadir, que en el presente } \\
\text { caso no sólo existe obligación } \\
\text { resarcitoria por la teorización } \\
\text { expuesta, sino que es tangible } \\
\text { negligencia en el proceder de la } \\
\text { demandada. }\end{array}$ & $\begin{array}{l}\text { FUNDAMENTOS JURIDICOS } \\
\text { PRIMERO.- Sobre la negligencia de la } \\
\text { demandada } \\
\text { Hay que [añadir] a las consideraciones } \\
\text { de la instancia, que (las cuales) hacemos } \\
\text { nuestras, que afirma (afirmando) la } \\
\text { responsabilidad de la codemandada } \\
\text { «Supermercado S. SA» y de (con } \\
\text { traslado de obligación indemnizatoria a } \\
\text { su compañía de) Seguros «B.P.S.R. SA», } \\
\text { tras analizar (con toma de decisión } \\
\text { basada en análisis de) la culpa desde un } \\
\text { punto de vista cuasi objetivo, y } \\
\text { obliga(ndo) a indemnizar a (aquel que) } \\
\text { quien saca un provecho derivado de } \\
\text { actividad empresarial (, bajo la premisa } \\
\text { de) cuando ocurre(ncia de un accidente } \\
\text { en la propiedad donde desarrolla el } \\
\text { negocio y siempre que no exista culpa } \\
\text { exclusiva de la víctima, (añadir), que en } \\
\text { el presente caso no sólo existe obligación } \\
\text { resarcitoria por (la teorización expuesta) } \\
\text { lo expuesto, sino que (es tangible) existe } \\
\text { negligencia en el proceder de la } \\
\text { demandada. }\end{array}$ \\
\hline $\begin{array}{l}\text { Literalizando documental obrante en } \\
\text { la alzada conteniendo carta de la } \\
\text { codemandada "Supermercado S. } \\
\text { SA» a su correduría de Seguros, en } \\
\text { relación con el accidente objeto de } \\
\text { análisis, «esta señora se tropezó con } \\
\text { unas cajas de mercancías que había } \\
\text { comprado otro cliente, justo a la } \\
\text { salida de nuestra escalera } \\
\text { mecánica», uniendo la testifical de } \\
\text { doña S.R.A., contestación a la } \\
\text { repregunta cuarta «presenció el } \\
\text { accidente, vio cómo pasaba la niña y } \\
\text { que la señora no podía pasar y se } \\
\text { cayó», es afirmable manifestar } \\
\text { negligencia en la demandada al no } \\
\text { tener en perfectas condiciones de } \\
\text { utilización la vía de salida del } \\
\text { establecimiento. }\end{array}$ & $\begin{array}{l}\text { SEGUNDO.- Omisión del deber de } \\
\text { cuidado } \\
\text { (Liberalizando) Dice literalmente la } \\
\text { documental (obrante) incorporada a los } \\
\text { autos en la alzada (conteniendo) que } \\
\text { contiene una carta de la codemandada } \\
\text { «Supermercado S. SA» a su correduría } \\
\text { de Seguros, en relación con el accidente } \\
\text { objeto de análisis, "esta señora se } \\
\text { tropezó con unas cajas de mercancías } \\
\text { que había comprado otro cliente, justo a } \\
\text { la salida de nuestra escalera mecánica»(, } \\
\text { uniendo). Como además la declaración } \\
\text { (la) testifical de doña S.R.A., } \\
\text { (contestación) al contestar a la } \\
\text { repregunta cuarta coincide en que } \\
\text { «presenció el accidente, vio cómo } \\
\text { pasaba la niña y que la señora no podía } \\
\text { pasar y se cayó», (es afirmable } \\
\text { manifestar) se puede apreciar la la }\end{array}$ \\
\hline
\end{tabular}




\begin{tabular}{|c|c|}
\hline & $\begin{array}{l}\text { negligencia (en) de la demandada al no } \\
\text { tener en perfectas condiciones de } \\
\text { utilización la vía de salida del } \\
\text { establecimiento. }\end{array}$ \\
\hline $\begin{array}{l}\text { Siendo la escalera mecánica, vía de } \\
\text { salida, de titularidad propia, siendo el } \\
\text { objeto, caja de mercancías, que } \\
\text { obstaculiza el final de la precitada } \\
\text { escalera propiedad de la demandada } \\
\text { siendo manifiesto que dicha vía debe } \\
\text { estar expedita, apta para su } \\
\text { utilización como salida del centro } \\
\text { comercial, no produciéndose tal } \\
\text { realidad, se plasma negligencia, } \\
\text { tangibilizada en la falta de actuación } \\
\text { de operario para retirar todo } \\
\text { obstáculo que impida la salida, y no } \\
\text { sólo la impida sino que cree riesgo } \\
\text { para las personas, materializado en } \\
\text { la presente, en el accidente } \\
\text { analizado, base de la reclamación. }\end{array}$ & $\begin{array}{l}\text { TERCERO.- Falta de diligencia del } \\
\text { demandado } \\
\text { (Siendo) Al ser la escalera mecánica(,) } \\
\text { propiedad del supermercado vía de } \\
\text { salida (, de titularidad propia), (siendo el } \\
\text { objeto,) al ser la caja de mercancías(,) } \\
\text { que obstaculiza el final de la (precitada) } \\
\text { escalera propiedad de la demandada, al } \\
\text { ser (siendo) manifiesto que dicha vía } \\
\text { debe estar expedita(,) y apta para su } \\
\text { utilización como salida del centro } \\
\text { comercial, y al no (produciéndose) } \\
\text { producirse tal realidad, se (plasma) } \\
\text { aprecia negligencia, (tangibilizada) } \\
\text { concretada en la falta de actuación de un } \\
\text { operario dedicado a (para) retirar (todo) } \\
\text { cualquier obstáculo que impida la } \\
\text { salida(,) y (no sólo la impida sino que) } \\
\text { cree un riesgo para las personas, } \\
\text { (materializado en la presente), como } \\
\text { sucede en el accidente analizado (, base } \\
\text { de la reclamación). }\end{array}$ \\
\hline $\begin{array}{l}\text { Por lo expuesto y lo reflejado en la } \\
\text { instancia, se desestima el recurso, } \\
\text { ratificándose la resolución recurrida e } \\
\text { imponiéndose a la apelante las } \\
\text { costas de la presente alzada por mor } \\
\text { del párrafo } 2 \text { del art. } 710 \text { de la } \\
\text { LECiv". }\end{array}$ & $\begin{array}{l}\text { QUINTO.- Costas } \\
\text { Por lo expuesto y lo reflejado en la } \\
\text { instancia, se desestima el recurso, } \\
\text { ratificándose la resolución recurrida y se } \\
\text { imponen (e imponiéndose) a la apelante } \\
\text { las costas de la presente alzada por mor } \\
\text { del párrafo } 2 \text { del art. } 710 \text { de la LECiv. }\end{array}$ \\
\hline
\end{tabular}

Tabla 1. Comparación de la sentencia original junto con la sentencia modificada

De este modo, una vez realizados todos los cambios propuestos, el resultado muestra una sentencia que, a nuestro juicio, puede ser comprendida sin problemas por un ciudadano medio y, por ende, cumple uno de los objetivos del Plan de Transparencia Judicial de ofrecer a los ciudadanos una justicia comprensible, con un lenguaje inteligible en las sentencias y demás resoluciones judiciales.

Vistas las dos sentencias en las lenguas española e inglesa y dadas las numerosas diferencias que existen entre ellas, resulta de interés para el 
traductor plantearse las siguientes cuestiones: ¿Cómo habría que traducir, por ejemplo, de español a inglés? ¿Sería conveniente mantener la estructura original o, por el contrario, emplear la organización del texto británica? Ambas cuestiones se abordarán en el siguiente apartado del presente artículo.

\subsection{Traducción de las sentencias británica y española}

Como se ha comprobado anteriormente, las sentencias española y británica distan de ser similares pues cada una de ellas muestra unos rasgos distintos. Por un lado, la sentencia británica analizada, en consonancia con lo recomendado por el Plain English, muestra una estructura, una sintaxis y un léxico fácilmente comprensible; por el otro lado, la sentencia española analizada se caracteriza por un lenguaje enrevesado, una sintaxis farragosa y una estructura confusa. En consecuencia, la traducción de la sentencia de español a inglés, de por sí compleja como toda traducción jurídica, entraña un ejercicio no exento de dificultad si uno de los propósitos es el de acercarnos a un lenguaje comprensible tal y como preconiza el Plan de Transparencia Judicial español.

De esta forma, en el caso de que se nos encomendase traducir la sentencia de español a inglés, en particular en un contexto pedagógico, proponemos seguir los siguientes pasos:

1. Llevar a cabo un proceso de documentación exhaustivo en aras a determinar con éxito la macroestructura adecuada del texto jurídico en cuestión. Para ello, puede ser de utilidad la base de datos jurídica Westlaw ${ }^{8}$, en la cual encontramos la jurisprudencia de prácticamente todos los tribunales de nuestro país. De esta forma, una vez hallada la estructura clásica de una sentencia española, es decir, los apartados antecedentes de hecho, fundamentos de derecho y el fallo, se procedería a traducir siguiendo la estructura británica con la división de párrafos y la numeración.

2. Tras haber determinado la estructura de la sentencia, antes de proceder a realizar la traducción interlingüística o traducción propiamente dicha, habría que comenzar con una traducción intralingüística o reformulación, de acuerdo con la terminología jakobsoniana (Jakobson, 1974: 69), ya que traduciríamos dentro de la misma lengua, en este caso, española, tal y como hemos hecho en el apartado precedente. Ello se debe a que la sentencia española que nos ocupa resulta casi incomprensible y, por tanto,

\footnotetext{
${ }^{8}$ La URL de la base de datos jurídica Westlaw es la siguiente: <http://www.westlaw.es/in dex_spa.html?brand=nwles $>$.
} 
es necesario, antes de traducir, comprender, tal y como apunta Hurtado Albir (1006: 43) al hablar de la competencia traductora. Por tanto, sería necesario llevar a cabo una traducción intralingüística y adaptar el texto al nivel de un ciudadano medio español para luego trasladarlo a un Plain English.

3. Una vez que se ha comprendido el texto origen en su totalidad, se procedería a su traducción a la lengua inglesa. En este proceso, la documentación desempeña un papel sumamente importante pues ha de emplearse para realizar las consultas terminológicas, culturales y de equivalencia pertinentes. En este punto, el empleo de diccionarios especializados como el Alcaraz Varó y Hughes (2003), de obras en torno a la traducción jurídica ${ }^{9}$ así como de páginas web con recursos jurídicos, como puede ser la del grupo de investigación Lexicografía y Traducción (HUM-106) ${ }^{10}$, del proyecto TURICOR ${ }^{11}$ o la del grupo Gentt (Géneros Textuales para la Traducción $)^{12}$ resulta imprescindible.

4. Tras haber traducido el texto de español a inglés, resulta preciso llevar a cabo un proceso de revisión o corrección en el que, de acuerdo con Rabadán y Fernández Nistal (2002: 34) «se procede a la [...] identificación de posibles errores que hayan de ser subsanados, verificación de que se ha cumplido el objetivo funcional y revisión de los aspectos físicos del TM».

De este modo, estimamos que la realización de las cuatro fases propuestas ayudaría en gran medida a conseguir una traducción satisfactoria que, en la medida de lo posible, habrá procurado traducir o adaptar el Plain English a un español jurídico-administrativo más comprensible y accesible para el ciudadano medio.

Sin embargo, somos conscientes de que hemos de considerar que una sentencia judicial, por su carácter pragmático ejecutivo, puede y suele manifestar distintas disposiciones textuales entre dos culturas (en este caso

\footnotetext{
${ }^{9}$ Sobre el lenguaje jurídico o judicial así como sobre su traducción véase Borja Albí (2000), Monzó y Borja Albí (2005), Mayoral Asensio (1999) y Monzó Nebot (2002). En torno a la problemática de la terminología jurídica y la traducción de documentos legales u oficiales han reflexionado, entre otros, Gémar (1979 y 1982), Sparer y Schwab (1980), Bauer-Bernet (1983), Weston (1983), Hickey (1993 y 1996), Álvarez (1994) y García Izquierdo (2005).

${ }^{10} \mathrm{La}$ URL del grupo Lexicografía y Traducción es la siguiente: <http://www.uma.es/hum106/>.

${ }^{11}$ Se trata del proyecto TURICOR: Compilación de un corpus de contratos turísticos (alemán, español, inglés, italiano) para la generación textual multilingüe y la traducción jurídica. Entidad financiadora: Ministerio de Ciencia y Tecnología (Plan Nacional de Investigación Científica, Desarrollo e Innovación Tecnológica) y Unión Europea (Fondos FEDER). № de ref. BFF200304616 (2003-2006), cuya URL es la siguiente: <http://www.turicor.com/>.

${ }^{12}$ La URL del grupo Gentt es la siguiente: <http://www.gentt.uji.es/>.
} 
la anglosajona y la hispánica europea) que, de transformarse en algún grado, ya no responden a sendas tradiciones textuales expositivas. En el fondo a lo que estamos aludiendo es al alcance ético de la labor traductora, ya que la conciencia lingüística del traductor puede exigir modificaciones del texto original en busca de su mejora expositiva, pero tal postura, con ser perfectamente lícita desde la perspectiva de la praxis traductora, puede ser muy contraproducente para el autor jurídico del texto debido a que se puede llegar a considerar desproporcionada o simplemente falsaria.

Además, dado que el traductor puede verse sometido a un juicio por parte del autor de los textos por considerar que se ha modificado a sabiendas el contenido y la forma de la sentencia, con lo cual, en parte, podría incurrir en una responsabilidad dolosa, estimamos oportuno que las modificaciones textuales propuestas para ciertos productos de la tradición jurídica hispánica sean convenientemente justificados ante el autor o autores de los textos, para evitar acusaciones infundadas de dolo o falsificación.

\section{CONCLUSIONES}

En el presente artículo hemos abordado el caso de la simplificación del discurso jurídico-administrativo en Gran Bretaña y en España. En cuanto al inglés jurídico, se advierte que el Plain English ha logrado calar en el lenguaje de la ley y de la administración de la justicia dado que ha influido en parte en la simplificación del lenguaje procesal de la nueva Ley de Enjuiciamiento Civil de 1998. Asimismo, dicha simplificación ha quedado patente en la sentencia del High Court of Justice por un caso de fraude, analizada en este artículo, cuya organizada macroestructura y asequible vocabulario muestran el deseo de alcanzar claridad y transparencia judicial.

Respecto a nuestro país, si bien las iniciativas para la modernización del discurso jurídico-administrativo no han comenzado hasta hace poco, cabe destacar en particular la aprobación en 2002 del Pacto de Estado para la Reforma de la Justicia, dentro del cual merece especial atención el Plan de Transparencia Judicial, aprobado en 2005, y que aboga por una mayor transparencia de los procedimientos judiciales y, por extensión, del español jurídico. No obstante, tras analizar una sentencia emitida por un Juzgado de Instancia, hemos constatado que el lenguaje empleado en la redacción de este documento dista sobremanera de ser un discurso inteligible y fácilmente comprensible para un lego en la materia. En consecuencia, concluimos que aún es preciso avanzar en la modernización y simplificación del discurso jurídico-administrativo español, pues, en comparación con el inglés, aún no ha alcanzado las características necesarias para definirlo como un Plain Spanish. 
Asimismo, esta falta de similitud en el lenguaje jurídico de las dos sentencias analizadas tiene implicaciones en la traducción español-inglés, ya que, en primer lugar, antes de comenzar a traducir de una lengua a otra, es decir, antes de realizar una traducción interlingüística, habría de llevarse a cabo una traducción intralingüística, esto es, adaptar la sentencia para que llegase a ser fácilmente comprensible. Además, la labor de documentación desempeña, en este caso, un papel sumamente importante, pues estaría presente en todo el proceso traductológico, en particular:

a) en la labor de documentación previa a la traducción en aras a recabar toda la información pertinente respecto a las estructuras de las sentencias en los dos ordenamientos jurídicos;

b) en la labor de documentación durante la fase onomasiológica del proceso y que implica búsquedas terminológicas, fraseológicas o conceptuales.

c) en la labor de documentación una vez realizada la traducción a fin de revisar y corregir el texto.

En suma, la simplificación y modernización del discurso jurídico-administrativo por la que abogan muchos ciudadanos y que se plasma en diversos movimientos y campañas, así como en diversas iniciativas por parte de los Estados, cobra cada vez mayor relevancia pues encarna el ideal de democracia y de igualdad ante la Justicia. No obstante, aún falta un largo camino por recorrer, en especial en España, pero, sin lugar a dudas, los avances son cada vez más patentes. En definitiva, tal y como se ha señalado:

La solución al problema es de integración y de equilibrio [...]. Romper con la inercia de esa expresión automática [...] puede ser una tarea nobilísima capaz de ilusionar a los juristas, si aceptan verla, como una vía de profundizar en la relación de nuestro Estado de Derecho, democrático y de cultura (Prieto 1996: 129).

En este punto, el traductor cobra importancia en el sentido de que, a la hora de enfrentarse a una traducción, debe reflejar este lenguaje llano y sencillo o, lo que es lo mismo, el Plain Spanish con una doble negociación, de todo punto imprescindible: a) relativa al traductor en relación con lo que tiene que traducir y su cultura receptora; b) relativa a la negociación, tal vez preventiva, con el propio autor del texto original. 
REFERENCIAS BIBLIOGRÁFICAS

AlCARAZ VARÓ, Enrique, El español jurídico. Barcelona: Ariel, 2002. , El inglés jurídico (5. ${ }^{\mathrm{a}}$ ed.). Barcelona: Ariel, 2001.

y HUGHES, Brian, Diccionario de términos jurídicos: inglés-español/español-inglés. Barcelona: Ariel, 1993. , Legal Translation Explained. Manchester: St. Jerome, 2002.

ÁlvAREZ, María Antonia, Traducción jurídica inglés-español. Madrid: Universidad Nacional de Educación a Distancia, 1994.

BAUER-BERNET, Hélène, Droit, informatique et traduction: l'expérience de la Communauté économique européenne. La puce et le dragon à sept langues. Montréal: Conseil de la langue française, 1983.

BORJA ALBI, Anabel, El texto jurídico inglés y su traducción al español. Barcelona: Ariel Lenguas Modernas, 2000.

CALvo Ramos, Luciana, Introducción al estudio del lenguaje administrativo: gramática y textos. Madrid: Gredos, 1980.

COMET I CODINA, Robert, "La modernització dels llenguatges administratius europeus". En Tradició i Modernitat en el Llenguatge Administratiu. Barcelona: E.A.P.C, 1986, pp. 23-46.

GARCÍA IZQUIERDO, Isabel, El género textual y la traducción: reflexiones teóricas y aplicaciones pedagógicas. Berna: Peter Lang, 2005.

GemAR, Jean-Claude, La traduction juridique. En Méta, 24, 1, 1979. , The Language of the Law and Translation: Essays on Jurilinguistics, Québec: Éditeur Officiel du Québec, 1986.

ETXEBARRÍA, Maitena, "El lenguaje jurídico-administrativo: propuestas para su modernización y normalización". En Revista Española de Lingüística, 27, 2, 1997, pp. 357-375.

HICKEY, Leo, "Equivalency, certainly but is it legal?". En Turjuman, 2, 2, 1993, pp. 65-76.

, "Aproximación didáctica a la traducción jurídica". En La enseñanza de la traducción. Castellón de la Plana: Publicaciones de la Universitat Jaume I, 1996. pp. 127-40.

HURTADO AlBIR, Amparo, "La enseñanza de la traducción directa 'general'. Objetivos de aprendizaje y metodología". En La enseñanza de la traducción. Castellón, Universitat Jaume I: Col. Estudis sobre la traducció, 3, 1996, pp. 31-55.

JAKOBSON, Roman, Ensayos de lingüística general. Barcelona: Seix Barral, 1974.

LERAT, Pierre, Las lenguas especializadas. Barcelona: Ariel, 1997.

Martín, Jacinto, Ruiz, Reyes, SANTAELla, Juan y EscánEZ, José, Los lenguajes especiales. Granada: Comares, 1996. 
Ministerio PARA LAS Administraciones PúBlicas (M.A.P.), Manual de Estilo del Lenguaje Administrativo, Madrid: INAP, 1990.

MAYORAL ASENSIO, Roberto, "Traducción oficial (jurada) y función". En Traducir para la justicia, Granada: Comares, 1999, pp. 59-86.

MONZÓ NEBOT, Esther, La professió del traductor juridic $i$ jurat: descripció sociológica del profesional $i$ anàlisi discursiva del transgènere, Castelló de la Plana: Universitat Jaume I, 2002.

MONZÓ NEBOT, Esther y BORJA ALBI, Anabel, La traducción y la interpretación en las relaciones jurídicas internacionales. Castelló de la Plana: Universitat Jaume I, 2005.

Plain English Campaign. Language on Trial, The Plain English Guide to Legal Writing. London: Robson Books, 1996.

Prieto de Pedro, Jesús, Lenguas, lenguaje y derecho. Madrid: Civitas, 1991.

, "La exigencia de un buen lenguaje jurídico y Estado de Derecho. Centro de Estudios y Constitucionales". En Revista de Administración Pública, 140, 1996, pp. 111-130.

RABADÁN, Rosa y FERNÁNDEZ NISTAL, Purificación, La traducción inglés-español: fundamentos, herramientas, aplicaciones. León: Servicio de publicaciones de la Universidad, 2002.

RODRíGUEZ ACHÚTEGUI, Edmundo, "El lenguaje de la sentencia y la exigencia de hechos probados en el orden jurisdiccional civil y contencioso administrativo". En Estudios de derecho judicial, 109, 2006, pp. 247-273.

SENTENCING GUIDELINES COUNCIL [en línea], "Theft and Burglary Guideline". $<$ http://www.sentencing-guidelines.gov.uk/> [Consulta: 15 febrero de 2011.]

SOLAN, Lawrence, The Languages of Judges. Chicago: The University of Chicago Press, 1993.

SPARER, Michel y SCHWAB, Wallace, Redaction des lois: rendez-vous du droit et de la culture. Montréal: Conseil de la langue française, 1980.

TOLEDO BÁEZ, María Cristina, "From Plain English to Plain Spanish?: Proposals to Modernise Administrative and Legal Language in Europe and Spain and its impact on Legal Translation". En P. SÁNCHEZ Hernández, P. Pérez-Paredes, P. Aguado Jiménez y R. Criado SÁNCHEZ (eds.). Researching and Teaching Specialized Languages: New Contexts, New Challenges. Murcia: Servicio de Publicaciones de la Universidad, 2008, pp. 450-460.

, El resumen automático como recurso documental para la traducción de artículos de investigación del ámbito jurídicotecnológico (español-inglés-francés). Málaga: Servicio de Publicaciones de la Universidad, 2009. 
El resumen automático y la evaluación de traducciones en el contexto de la traducción especializada. Frankfurt: Peter Lang, 2010.

Weston, Martin, "Problems and Principles in Legal Translation". En The Incorporated Linguist, 22, 4, 1983. pp. 207-211. 